Bull. Austral. Math. Soc.

VoL. 46 (1992) [115-125]

\title{
CHARACTERISATIONS FOR ANALYTIC FUNCTIONS OF BOUNDED MEAN OSCILLATION
}

\author{
JiE MiaO
}

Let $\alpha>0$ and let $f^{[\alpha]}(z)$ be the $\alpha$ th fractional derivative of an analytic function $f$ on the unit disc $D$. In this paper we show that $f \in$ BMOA if and only if $\left|f^{[\alpha]}(z)\right|^{2}\left(1-|z|^{2}\right)^{2 \alpha-1} d A(z)$ is a Carleson measure and $f \in$ VMOA if and only if $\left|f^{[\alpha]}(z)\right|^{2}\left(1-|z|^{2}\right)^{2 \alpha-1} d A(z)$ is a vanishing Carleson measure, where $A$ denotes the normalised Lebesgue measure on $D$. Hence a significant extension of familiar characterisations for analytic functions of bounded and vanishing mean oscillation is obtained.

\section{INTRODUCTION}

Let $D=\{z:|z|<1\}$. The space of analytic functions on $D$ of bounded mean oscillation, denoted by BMOA, consists of all functions in $H^{2}$ for which

$$
\sup _{\lambda \in D}\left(\frac{1}{2 \pi} \int_{0}^{2 \pi}\left|f\left(\varphi_{\lambda}\left(e^{i \theta}\right)\right)-f(\lambda)\right|^{2} d \theta\right)^{1 / 2}<\infty
$$

where $\varphi_{\lambda}(z)=(z-\lambda) /(1-\bar{\lambda} z)$. BMOA is a Banach space under the norm given by

$$
\|f\|_{\mathrm{BMOA}}=|f(0)|+\sup _{\lambda \in D}\left(\frac{1}{2 \pi} \int_{0}^{2 \pi}\left|f\left(\varphi_{\lambda}\left(e^{i \theta}\right)\right)-f(\lambda)\right|^{2} d \theta\right)^{1 / 2} .
$$

VMOA, the subspace of BMOA, consists of all functions $f$ in $H^{2}$ for which

$$
\lim _{|\lambda| \rightarrow 1-0} \int_{0}^{2 \pi}\left|f\left(\varphi_{\lambda}\left(e^{i \theta}\right)\right)-f(\lambda)\right|^{2} d \theta=0 .
$$

It is well known that $f \in \mathrm{BMOA}$ if and only if

$$
\sup _{\lambda \in D}\left(\int_{D}\left|f^{\prime}(z)\right|^{2}\left(1-\left|\varphi_{\lambda}(z)\right|^{2}\right) d A(z)\right)^{1 / 2}<\infty
$$

Received 2 July 1991

This research was partially supported by a grant from NSF of P.R. China.

Copyright Clearance Centre, Inc. Serial-fee code: 0004-9729/92 \$A2.00+0.00. 
and $f \in$ VMOA if and only if

$$
\lim _{|\lambda| \rightarrow 1-0} \int_{D}\left|f^{\prime}(z)\right|^{2}\left(1-\left|\varphi_{\lambda}(z)\right|^{2}\right) d A(z)=0
$$

where $A$ denotes the normalised Lebesgue area measure on $D$. In other words, $f \in$ BMOA if and only if $\left|f^{\prime}(z)\right|^{2}\left(1-|z|^{2}\right) d A(z)$ is a Carleson measure on $D$, and $f \in$ VMOA if and only if $\left|f^{\prime}(z)\right|^{2}\left(1-|z|^{2}\right) d A(z)$ is a vanishing Carleson measure on $D$. The definitions of the Carleson measure and the vanishing Carleson measure can be found in $[1,2,3]$.

In this paper we consider fractional derivatives. Let $f(z)=\sum_{n=0}^{\infty} a_{n} z^{n}$ be analytic on $D$ and $\alpha>0$, the $\alpha$ th fractional derivative of $f$ is defined by

$$
f^{[\alpha]}(z)=\sum_{n=0}^{\infty} \frac{\Gamma(n+1+\alpha)}{n !} a_{n} z^{n}
$$

and $f^{[\alpha]}(z)$ is analytic on $D$. We allow fractional derivatives of $f$ to appear in place of $f^{\prime}$ in the integral on the left of (1) and (2). Hence a significant extension of the two characterisations for BMOA and VMOA is obtained.

For our purpose let us recall the definition of the Bloch space. The Bloch space $\mathcal{B}$ consists of all analytic functions on $D$ for which $\sup _{z \in D}\left(1-|z|^{2}\right)\left|f^{\prime}(z)\right|<\infty$. B is a Banach space under the norm given by

$$
\|f\|_{B}=|f(0)|+\sup _{z \in D}\left(1-|z|^{2}\right)\left|f^{\prime}(z)\right|
$$

The little Bloch space $\mathcal{B}_{0}$, a subspace of $\mathcal{B}$, consists of all analytic functions on $D$ for which $\lim _{|z| \rightarrow 1-0}\left(1-|z|^{2}\right)\left|f^{\prime}(z)\right|=0$. It is known (see [5]) that BMOA is contained in $\mathcal{B}$ and VMOA in $\mathcal{B}_{0}$, and there is a constant $C$ such that

$$
\|f\|_{B} \leqslant C\|f\|_{\mathrm{BMOA}}
$$

Throughout this paper $C$ denotes a positive constant, not necessarily the same at each occurrence, it is independent of $f$ and $\lambda$.

\section{EXTENSION OF CHARACTERISATIONS}

Our main result is the following theorem. 
THEOREM . Let $\alpha>0$ and let $f$ be an analytic function on $D$. Then

(I) $f \in B M O A$ if and only if

$$
\sup _{\lambda \in D}\left(\int_{D}\left|f^{[\alpha]}(z)\right|^{2}\left(1-|z|^{2}\right)^{2(\alpha-1)}\left(1-\left|\varphi_{\lambda}(z)\right|^{2}\right) d A(z)\right)^{1 / 2}<\infty
$$

(II) $f \in V M O A$ if and only if

$$
\lim _{|\lambda| \rightarrow 1-0} \int_{D}\left|f^{[\alpha]}(z)\right|^{2}\left(1-|z|^{2}\right)^{2(\alpha-1)}\left(1-\left|\varphi_{\lambda}(z)\right|^{2}\right) d A(z)=0 .
$$

The proof of the theorem is based on three propositions. After these results are established, the conclusions of the theorem are easy to obtain.

Proposition 1. For an analytic function $f$ on $D$ the following two quantities are equivalent:

(i) $\int_{D}\left|f^{\prime}(z)\right|^{2}\left(1-\left|\varphi_{\lambda}(z)\right|^{2}\right) d A(z)+\left(1-|\lambda|^{2}\right)|f(0)|^{2}$,

(ii) $\int_{D}\left|f^{[1]}(z)\right|^{2}\left(1-\left|\varphi_{\lambda}(z)\right|^{2}\right) d A(z)$.

Proof: A straightforward computation gives

$$
1-\left|\varphi_{\lambda}(z)\right|^{2}=\frac{\left(1-|\lambda|^{2}\right)\left(1-|z|^{2}\right)}{|1-\bar{\lambda} z|^{2}}, \quad(\lambda, z \in D) .
$$

Let $f(z)=\sum_{n=0}^{\infty} a_{n} z^{n}$. Then $f^{\prime}(z)=\sum_{n=1}^{\infty} n a_{n} z^{n-1}$ and $f^{[1]}(z)=\sum_{n=0}^{\infty}(n+1) a_{n} z^{n}$. Since $1 /(1-\bar{\lambda} z)=\sum_{n=0}^{\infty} \bar{\lambda}^{n} z^{n}$, we set

$$
f^{[1]}(z) \frac{1}{1-\bar{\lambda} z}=\sum_{n=0}^{\infty} b_{n} z^{n} \quad \text { and } \quad f^{\prime}(z) \frac{1}{1-\bar{\lambda} z}=\sum_{n=0}^{\infty} c_{n} z^{n}
$$

where $b_{n}=\sum_{i+j=n}(i+1) a_{i} \bar{\lambda}^{j}$ and $c_{n}=\sum_{i+j=n}(i+1) a_{i+1} \bar{\lambda}^{j}$. Now Parseval's formula shows that

$$
\int_{D}\left|f^{\prime}(z)\right|^{2} \frac{1-|z|^{2}}{|1-\bar{\lambda} z|^{2}} d A(z)=\sum_{n=0}^{\infty} \frac{\left|c_{n}\right|^{2}}{(n+1)(n+2)}
$$

and

$$
\int_{D}\left|f^{[1]}(z)\right|^{2} \frac{1-|z|^{2}}{|1-\bar{\lambda} z|^{2}} d A(z)=\sum_{n=0}^{\infty} \frac{\left|b_{n}\right|^{2}}{(n+1)(n+2)}
$$


We can suppose that $\lambda \neq 0$. It is easy to see the equivalence for $\lambda=0$. It follows from $b_{n} / \bar{\lambda}^{n}=\sum_{\lambda=0}^{n}(i+1) a_{i} / \bar{\lambda}^{i}$ that $a_{n} / \bar{\lambda}^{n}=(1 /(n+1))\left(b_{n} / \bar{\lambda}^{n}-b_{n-1} / \bar{\lambda}^{n-1}\right)$ for $n \geqslant 1$.

Thus for $n \geqslant 0$ we have

$$
\begin{aligned}
c_{n}=\bar{\lambda}^{n+1} \sum_{i=1}^{n+1} \frac{i a_{i}}{\bar{\lambda}^{i}} & =\bar{\lambda}^{n+1} \sum_{i=1}^{n+1} \frac{i}{i+1}\left(\frac{b_{i}}{\bar{\lambda}^{i}}-\frac{b_{i-1}}{\bar{\lambda}^{i-1}}\right) \\
& =\frac{n+1}{n+2} b_{n+1}-\bar{\lambda}^{n+1} \sum_{i=0}^{n} \frac{1}{(i+1)(i+2)} \frac{b_{i}}{\bar{\lambda}^{i}} .
\end{aligned}
$$

Similarly for $n \geqslant 2$ we have

$$
\begin{aligned}
b_{n}=\bar{\lambda}^{n} \sum_{i=0}^{n} \frac{(i+1) a_{i}}{\bar{\lambda}^{i}} & =\bar{\lambda}^{n} \sum_{i=2}^{n} \frac{i+1}{i}\left(\frac{c_{i-1}}{\bar{\lambda}^{i}}-\frac{c_{i-2}}{\bar{\lambda}^{i-1}}\right)+2 a_{1} \bar{\lambda}^{n-1}+a_{0} \bar{\lambda}^{n} \\
& =\frac{n+1}{n} c_{n-1}+\bar{\lambda}^{n} \sum_{i=0}^{n-2} \frac{1}{(i+1)(i+2)} \frac{c_{i}}{\bar{\lambda}^{i+1}}+a_{0} \bar{\lambda}^{n},
\end{aligned}
$$

$b_{0}=a_{0}$ and $b_{1}=2 C_{0}+a_{0} \bar{\lambda}$. First we show that quantity (i) is dominated by quantity (ii). By Hölder's inequality it follows that

$$
\left|\bar{\lambda}^{n+1} \sum_{i=0}^{n} \frac{1}{(i+1)(i+2)} \frac{b_{i}}{\bar{\lambda}^{i}}\right|^{2} \leqslant C \sum_{i=0}^{n} \frac{\left|b_{i}\right|^{2}}{(i+1)^{2}} .
$$

Hence

$$
\begin{aligned}
\sum_{n=0}^{\infty} \frac{\left|c_{n}\right|^{2}}{(n+1)(n+2)}+|f(0)|^{2} & \leqslant \sum_{n=0}^{\infty} \frac{2}{(n+1)(n+2)}\left(\left|b_{n+1}\right|^{2}+C \sum_{i=0}^{n} \frac{\left|b_{i}\right|^{2}}{(i+1)^{2}}\right)+\left|b_{0}\right|^{2} \\
& \leqslant C\left(\sum_{n=0}^{\infty} \frac{\left|b_{n}\right|^{2}}{(n+1)^{2}}+\sum_{i=0}^{\infty} \frac{\left|b_{i}\right|^{2}}{(i+1)^{2}} \sum_{n=i}^{\infty} \frac{1}{(n+1)(n+2)}\right) \\
& \leqslant C \sum_{n=0}^{\infty} \frac{\left|b_{n}\right|^{2}}{(n+1)^{2}} .
\end{aligned}
$$

For the converse, we use the same method to get

$$
\begin{aligned}
\sum_{n=0}^{\infty} \frac{\left|b_{n}\right|^{2}}{(n+1)(n+2)} & \leqslant C \sum_{n=2}^{\infty} \frac{1}{(n+1)^{2}}\left(\left|c_{n-1}\right|^{2}+\sum_{i=0}^{n-2} \frac{\left|c_{i}\right|^{2}}{(i+1)^{2}}+\left|c_{0}\right|^{2}+\left|a_{0}\right|^{2}\right) \\
& \leqslant C\left(\sum_{n=0}^{\infty} \frac{\left|c_{n}\right|^{2}}{(n+1)^{2}}+|f(0)|^{2}\right) .
\end{aligned}
$$

This completes the proof of the proposition. 
REMARK. By Proposition 1 we actually have

$$
\|f\|_{\mathrm{BMOA}} \approx \sup _{\lambda \in D}\left(\int_{D}\left|f^{[1]}(z)\right|^{2}\left(1-\left|\varphi_{\lambda}(z)\right|^{2}\right) d A(z)\right)^{1 / 2},
$$

and $f \in$ VMOA if and only if

$$
\lim _{|\lambda| \rightarrow 1-0} \int_{D}\left|f^{[1]}(z)\right|^{2}\left(1-\left|\varphi_{\lambda}(z)\right|^{2}\right) d A(z)=0 .
$$

The following result is a main step to prove the Theorem.

Proposition 2. Let $f$ be an analytic function on $D$.

(i) If $\alpha>0$, then

$$
\begin{aligned}
& \int_{D}\left|f^{[1]}(z)\right|^{2}\left(1-\left|\varphi_{\lambda}(z)\right|^{2}\right) d A(z) \\
& \quad \leqslant C \int_{D}\left|f^{[\alpha]}(z)\right|^{2}\left(1-|z|^{2}\right)^{2(\alpha-1)}\left(1-\left|\varphi_{\lambda}(z)\right|^{2}\right) d A(z) .
\end{aligned}
$$

(ii) If $\alpha>1 / 2$, then

$$
\begin{gathered}
\int_{D}\left|f^{[\alpha]}(z)\right|^{2}\left(1-|z|^{2}\right)^{2(\alpha-1)}\left(1-\left|\varphi_{\lambda}(z)\right|^{2}\right) d A(z) \\
\leqslant C \int_{D}\left|f^{[1]}(z)\right|^{2}\left(1-\left|\varphi_{\lambda}(z)\right|^{2}\right) d A(z)
\end{gathered}
$$

If $0<\alpha \leqslant 1 / 2$, then

$$
\begin{aligned}
& \int_{D}\left|f^{[\alpha]}(z)\right|^{2}\left(1-|z|^{2}\right)^{2(\alpha-1)}\left(1-\left|\varphi_{\lambda}(z)\right|^{2}\right) d A(z) \\
& \leqslant C\left(\int_{D}\left|f^{[1]}(z)\right|^{2}\left(1-\left|\varphi_{\lambda}(z)\right|^{2}\right) d A(z)\right. \\
&\left.+\int_{D}\left|f^{[1]}(z)\right|^{2} \frac{\left(1-|z|^{2}\right)^{2-2(\alpha-\beta)}\left(1-|\lambda|^{2}\right)^{2(\alpha-\beta)}}{|1-\bar{\lambda} z|^{2}} d A(z)\right),
\end{aligned}
$$

for all $\beta \in(0, \alpha)$.

Proof: Let $f(z)=\sum_{n=0}^{\infty} a_{n} z^{n}$. Then $f^{[\alpha]}(z)=\sum_{n=0}^{\infty}(\Gamma(n+1+\alpha) / n !) a_{n} z^{n}$. We set

$$
f^{[1]}(z) \frac{1}{1-\bar{\lambda} z}=\sum_{n=0}^{\infty} b_{n} z^{n} \quad \text { and } \quad f^{[\alpha]}(z) \frac{1}{1-\bar{\lambda} z}=\sum_{n=0}^{\infty} d_{n} z^{n}
$$


where $d_{n}=\sum_{i+j=n}(\Gamma(i+1+\alpha) / i !) a_{i} \bar{\lambda}^{j}$ and $b_{n}$ is the same as written in the proof of Proposition 1. It follows from (4) and Parseval's formula that

(9)

$$
\int_{D}\left|f^{[\alpha]}(z)\right|^{2}\left(1-|z|^{2}\right)^{2(\alpha-1)}\left(1-\left|\varphi_{\lambda}(z)\right|^{2}\right) d A(z)=\left(1-|\lambda|^{2}\right) \sum_{n=0}^{\infty} B(n+1,2 \alpha)\left|d_{n}\right|^{2},
$$

where $B(n+1,2 \alpha)=2 \int_{0}^{1} r^{2 n}\left(1-r^{2}\right)^{2 \alpha-1} r d r=(\Gamma(2 \alpha) \Gamma(n+1)) / \Gamma(n+1+2 \alpha)$. We can also suppose that $\lambda \neq 0$. Then as in the proof of Proposition 1 , for $n \geqslant 1$ we have

$$
\begin{aligned}
b_{n} & =\bar{\lambda}^{n} a_{0}+\bar{\lambda}^{n} \sum_{i=1}^{n} \frac{(i+1) !}{\Gamma(i+1+\alpha)}\left(\frac{d_{i}}{\bar{\lambda}^{i}}-\frac{d_{i-1}}{\bar{\lambda}^{i-1}}\right) \\
& =(\alpha-1) \bar{\lambda}^{n} \sum_{i=0}^{n-1} \frac{(i+1) !}{\Gamma(i+2+\alpha)} \frac{d_{i}}{\bar{\lambda}^{i}}+\frac{(n+1) !}{\Gamma(n+1+\alpha)} d_{n}
\end{aligned}
$$

on the other hand, for $n \geqslant 1$ we have

$$
\begin{aligned}
d_{n} & =\bar{\lambda}^{n} \Gamma(1+\alpha) a_{0}+\bar{\lambda}^{n} \sum_{i=1}^{n} \frac{\Gamma(i+1+\alpha)}{(i+1) !}\left(\frac{b_{i}}{\bar{\lambda}^{i}}-\frac{b_{i-1}}{\bar{\lambda}^{i-1}}\right) \\
& =(1-\alpha) \bar{\lambda}^{n} \sum_{i=0}^{n-1} \frac{\Gamma(i+1+\alpha)}{(i+2) !} \frac{b_{i}}{\bar{\lambda}^{i}}+\frac{\Gamma(n+1+\alpha)}{(n+1) !} b_{n}
\end{aligned}
$$

and $d_{0}=\Gamma(1+\alpha) b_{0}$. By Stirling's formula we know that $\Gamma(n+1+\alpha) / n ! \approx(n+1)^{\alpha}$, $B(n+1,2 \alpha) \approx(n+1)^{-2 \alpha}$. Using this fact and Hölder's inequality we get

$$
\begin{aligned}
\left|b_{n}\right|^{2} & \leqslant C\left((n+1)^{-2 \alpha+2}\left|d_{n}\right|^{2}+\left(\sum_{i=0}^{n}(i+1)^{-\alpha}\left|d_{i}\right|\right)^{2}\right) \\
& \leqslant C\left((n+1)^{-2 \alpha+2}\left|d_{n}\right|^{2}+\left(\sum_{i=0}^{n}(i+1)^{-1 / 2}\right)\left(\sum_{i=0}^{n}(i+1)^{-2 \alpha+1 / 2}\left|d_{i}\right|^{2}\right)\right) \\
& \leqslant C\left((n+1)^{-2 \alpha+2}\left|d_{n}\right|^{2}+(n+1)^{1 / 2} \sum_{i=0}^{n}(i+1)^{-2 \alpha+1 / 2}\left|d_{i}\right|^{2}\right)
\end{aligned}
$$


thus

$$
\begin{aligned}
& \sum_{n=0}^{\infty} \frac{\left|b_{n}\right|^{2}}{(n+1)(n+2)} \\
& \leqslant C\left(\sum_{n=0}^{\infty}(n+1)^{-2 \alpha}\left|d_{n}\right|^{2}+\sum_{n=0}^{\infty}(n+1)^{-3 / 2} \sum_{i=0}^{n}(i+1)^{-2 \alpha+1 / 2}\left|d_{i}\right|^{2}\right) \\
& \quad \leqslant C\left(\sum_{n=0}^{\infty}(n+1)^{-2 \alpha}\left|d_{n}\right|^{2}+\sum_{i=0}^{\infty}(i+1)^{-2 \alpha+1 / 2}\left|d_{i}\right|^{2} \sum_{n=i}^{\infty}(n+1)^{-3 / 2}\right) \\
& \quad \leqslant C \sum_{n=0}^{\infty}(n+1)^{-2 \alpha}\left|d_{n}\right|^{2} \leqslant C \sum_{n=0}^{\infty} B(n+1,2 \alpha)\left|d_{n}\right|^{2} .
\end{aligned}
$$

In view of (6) and (9), this proves (i) of the proposition.

To prove (ii), we treat the case $\alpha>1 / 2$ and $\alpha \leqslant 1 / 2$ separately. Given $\alpha>0$, we can choose a positive number $\beta$ small enough such that $\alpha-\beta>1 / 2$ for $\alpha>1 / 2$, and $\alpha-\beta>0$ for $0<\alpha \leqslant 1 / 2$. By the same reason we have

$$
\begin{aligned}
\left|d_{n}\right|^{2} & \leqslant C\left((n+1)^{2 \alpha-2}\left|b_{n}\right|^{2}+|\lambda|^{2 n}\left(\sum_{i=0}^{n}(i+1)^{\alpha-2} \frac{\left|b_{i}\right|}{|\lambda|^{i}}\right)^{2}\right) \\
& \leqslant C\left((n+1)^{2 \alpha-2}\left|b_{n}\right|^{2}+|\lambda|^{2 n}\left(\sum_{i=0}^{n}(i+1)^{2 \beta-1}\right)\left(\sum_{i=0}^{n}(i+1)^{2(\alpha-\beta)-3} \frac{\left|b_{i}\right|^{2}}{|\lambda|^{2 i}}\right)\right) \\
& \leqslant C\left((n+1)^{2 \alpha-2}\left|b_{n}\right|^{2}+|\lambda|^{2 n}(n+1)^{2 \beta} \sum_{i=0}^{n}(i+1)^{2(\alpha-\beta)-3} \frac{\left|b_{i}\right|^{2}}{|\lambda|^{2 i}}\right) .
\end{aligned}
$$

The case $\alpha>1 / 2$ is easier:

$$
\begin{aligned}
& \sum_{n=0}^{\infty} B(n+1,2 \alpha)\left|b_{n}\right|^{2} \\
& \leqslant C\left(\sum_{n=0}^{\infty}(n+1)^{-2}\left|b_{n}\right|^{2}+\sum_{n=0}^{\infty}(n+1)^{-2 \alpha+2 \beta} \sum_{i=0}^{n}(i+1)^{2(\alpha-\beta)-s}\left|b_{i}\right|^{2}\right) \\
& \quad=C\left(\sum_{n=0}^{\infty}(n+1)^{-2}\left|b_{n}\right|^{2}+\sum_{i=0}^{\infty}(i+1)^{2(\alpha-\beta)-3}\left|b_{i}\right|^{2} \sum_{n=i}^{\infty}(n+1)^{-2 \alpha+2 \beta}\right) \\
& \quad \leqslant C \sum_{n=0}^{\infty}(n+1)^{-2}\left|b_{n}\right|^{2},
\end{aligned}
$$

since $-2 \alpha+2 \beta<-1$. The first part of (ii) is proved. 
For $\alpha \leqslant 1 / 2$, the above method fails to work, so we need to modify the argument. It is clear that we need only consider the following term:

$$
\begin{aligned}
S_{n} & =|\lambda|^{2 n}(n+1)^{2 \beta} \sum_{i=0}^{n}(i+1)^{2(\alpha-\beta)-3} \frac{\left|b_{i}\right|^{2}}{|\lambda|^{2 i}} \\
& \leqslant|\lambda|^{2 n}(n+1)^{2 \beta}\left(\sum_{i=0}^{[n / 2]}(i+1)^{2(\alpha-\beta)-3} \frac{\left|b_{i}\right|^{2}}{|\lambda|^{2 i}}+\sum_{i=[n / 2]}^{n}(i+1)^{2(\alpha-\beta)-3} \frac{\left|b_{i}\right|^{2}}{|\lambda|^{2 i}}\right) \\
& \leqslant|\lambda|^{n}(n+1)^{2 \beta} \sum_{i=0}^{[n / 2]}(i+1)^{2(\alpha-\beta)-3}\left|b_{i}\right|^{2}+(n+1)^{2 \beta} \sum_{i=[n / 2]}^{n}(i+1)^{2(\alpha-\beta)-3} \frac{\left|b_{i}\right|^{2}}{|\lambda|^{2 i}} .
\end{aligned}
$$

Hence

(11)

$$
\begin{aligned}
& \sum_{n=0}^{\infty} B(n+1,2 \alpha) S_{n} \\
& \leqslant C\left(\sum_{n=0}^{\infty}(n+1)^{-2 \alpha+2 \beta}|\lambda|^{n} \sum_{i=0}^{[n / 2]}(i+1)^{2(\alpha-\beta)-3}\left|b_{i}\right|^{2}\right. \\
&\left.+\sum_{n=0}^{\infty}(n+1)^{-2 \alpha+2 \beta} \sum_{i=[n / 2]}^{n}(i+1)^{2(\alpha-\beta)-3}\left|b_{i}\right|^{2}\right) \\
& \leqslant\left(\left(\sum_{n=0}^{\infty}(n+1)^{-2 \alpha+2 \beta}|\lambda|^{n}\right)\left(\sum_{i=0}^{\infty}(i+1)^{2(\alpha-\beta)-3}\left|b_{i}\right|^{2}\right)\right. \\
&\left.\left.\left.+\sum_{i=0}^{\infty}(i+1)^{2(\alpha-\beta)-3}\left|b_{i}\right|^{2} \sum_{n=i}^{2 i+1}(n+1)^{-2 \alpha+2 \beta}\right)^{\infty}\right)_{n=0}^{\infty}(n+1)^{2(\alpha-\beta)}\left|b_{i}\right|^{2}+\sum_{n=0}^{\infty}(n+1)^{-2}\left|b_{n}\right|^{2}\right) \\
& \leqslant\left((1-|\lambda|)^{2(\alpha-\beta)-1} \sum_{n=0}^{\infty}(n)\right.
\end{aligned}
$$

where we used the fact that $\sum_{n=0}^{\infty}(n+1)^{\gamma}|\lambda|^{n} \leqslant C(1-|\lambda|)^{-\gamma-1}$ for $\gamma>-1$ in the last step. Again Parseval's formula and Stirling's formula show that

$$
\sum_{n=0}^{\infty}(n+1)^{2(\alpha-\beta)-3}\left|b_{i}\right|^{2} \approx \int_{D}\left|f^{[1]}(z)\right|^{2} \frac{\left(1-|z|^{2}\right)^{2-2(\alpha-\beta)}}{|1-\bar{\lambda} z|^{2}} d A(z) .
$$

Combining (10), (11) and (12) shows that the second part of (ii) holds. This completes the proof.

To give the third proposition, we need two lemmas. 
LEMMA 1. Let $f$ be an analytic function on $D$.

(i) If $f \in \mathcal{B}$, then $\sup _{z \in D}\left(1-|z|^{2}\right)\left|f^{(1)}(z)\right| \leqslant C\|f\|_{B}$.

(ii) If $f \in \mathcal{B}_{0}$, then $\lim _{|z| \rightarrow 1-0}\left(1-|z|^{2}\right)\left|f^{[1]}(z)\right|=0$.

Proof: Since $f^{[1]}(z)=f(z)+z f^{\prime}(z)$, we have

$$
\left|f^{[1]}(z)\right| \leqslant|f(z)|+\left|f^{\prime}(z)\right| \text {. }
$$

For $f \in \mathcal{B}$, we get

$$
\begin{aligned}
\left(1-|z|^{2}\right)|f(z)| & =\left(1-|z|^{2}\right)\left|\int_{0}^{z} f^{\prime}(\lambda) d \lambda+f(0)\right| \\
& \leqslant\left(1-|z|^{2}\right)\left(\int_{0}^{|z|}\left|f^{\prime}(\lambda)\right| d|\lambda|+|f(0)|\right) \\
& \leqslant\|f\|_{B}\left(1-|z|^{2}\right) \int_{0}^{|z|} \frac{d|\lambda|}{1-|\lambda|^{2}}+\left(1-|z|^{2}\right)|f(0)| \\
& \leqslant\|f\|_{B}\left(1-|z|^{2}\right) \log \frac{1}{1-|z|}+\left(1-|z|^{2}\right)|f(0)| .
\end{aligned}
$$

Then statements (i) and (ii) follow at once from the above inequalities and the definitions of the spaces $\mathcal{B}$ and $\mathcal{B}_{0}$.

LEMMA 2. $[4, \mathrm{p} .291]$ If $\gamma>-1$ and $m>1+\gamma$, then for $0 \leqslant \rho<1$,

$$
\int_{0}^{1}(1-\rho r)^{-m}(1-r)^{\gamma} d r \leqslant C(1-\rho)^{1+\gamma-m}
$$

Proposition 3. Let $0<\alpha \leqslant 1 / 2,0<\beta<\alpha$ and let $f$ be an analytic function on $D$.

(i) If $f \in \mathcal{B}$, then

$$
\sup _{\lambda \in D} \int_{D}\left|f^{[1]}(z)\right|^{2} \frac{\left(1-|z|^{2}\right)^{2-2(\alpha-\beta)}\left(1-|\lambda|^{2}\right)^{2(\alpha-\beta)}}{|1-\bar{\lambda} z|^{2}} d A(z) \leqslant C\|f\|_{B}^{2} .
$$

(ii) If $f \in \mathcal{B}_{0}$, then

$$
\lim _{|\lambda| \rightarrow 1-0} \int_{D}\left|f^{[1]}(z)\right|^{2} \frac{\left(1-|z|^{2}\right)^{2-2(\alpha-\beta)}\left(1-|\lambda|^{2}\right)^{2(\alpha-\beta)}}{|1-\bar{\lambda} z|^{2}} d A(z)=0 .
$$


Proof: For $f \in \mathcal{B}$, using Lemmas 1 and 2, we have

$$
\begin{aligned}
\int_{D}\left|f^{[1]}(z)\right|^{2} & \frac{\left(1-|z|^{2}\right)^{2-2(\alpha-\beta)}\left(1-|\lambda|^{2}\right)^{2(\alpha-\beta)}}{|1-\bar{\lambda} z|^{2}} d A(z) \\
& \leqslant C\|f\|_{B}^{2} \int_{D} \frac{\left(1-|z|^{2}\right)^{-2(\alpha-\beta)}\left(1-|\lambda|^{2}\right)^{2(\alpha-\beta)}}{|1-\bar{\lambda} z|^{2}} d A(z) \\
& \leqslant C\|f\|_{B}^{2}\left(1-|\lambda|^{2}\right)^{2(\alpha-\beta)} \int_{0}^{1}(1-r)^{-2(\alpha-\beta)}(1-|\lambda| r)^{-1} d r \\
& \leqslant C\|f\|_{B}^{2}\left(1-|\lambda|^{2}\right)^{2(\alpha-\beta)}(1-|\lambda|)^{-2(\alpha-\beta)} \leqslant C\|f\|_{B}^{2} .
\end{aligned}
$$

Thus statement (i) is proved.

If $f \in \mathcal{B}_{0}$, according to Lemma 1 (ii), for any $\varepsilon>0$, we can choose a positive number $\rho$ sufficiently close to 1 such that $\left(1-|z|^{2}\right)\left|f^{[1]}(z)\right|<\varepsilon$ for $\rho<|z|<1$. Then

$$
\int_{\rho<|z|<1}\left|f^{[1]}(z)\right|^{2} \frac{\left(1-|z|^{2}\right)^{2-2(\alpha-\beta)}\left(1-|\lambda|^{2}\right)^{2(\alpha-\beta)}}{|1-\bar{\lambda} z|^{2}} d A(z)<C \varepsilon^{2}
$$

On the other hand,

$$
\begin{aligned}
\int_{|z| \leqslant \rho}\left|f^{[1]}(z)\right|^{2} & \frac{\left(1-|z|^{2}\right)^{2-2(\alpha-\beta)}\left(1-|\lambda|^{2}\right)^{2(\alpha-\beta)}}{|1-\bar{\lambda} z|^{2}} d A(z) \\
& \leqslant C\|f\|_{B}^{2}\left(1-|\lambda|^{2}\right)^{2(\alpha-\beta)} \int_{0}^{\rho}(1-r)^{-2(\alpha-\beta)-1} d r .
\end{aligned}
$$

Now $\rho$ is fixed and if $1-|\lambda|$ is sufficiently small, then the last quantity can be less than or equal to $C\|f\|_{B}^{2} \varepsilon$. Combining the above inequalities shows that (ii) holds. This completes the proof of the proposition.

Proof of Theorem: By Proposition 2 (i), (7) and (8) it is easy to see that the conditions in (I) and (II) are sufficient for containment in BMOA and VMOA, respectively.

The necessity part of (I) follows immediately from Proposition 2 (ii), (7), Proposition 3 (i) and (3). The necessity part of (II) follows immediately from Proposition 2 (ii), (8) and Proposition 3 (ii). This completes the proof.

Finally, according to familiar characterisations of Carleson measures and vanishing Carleson measures (see for example [1, Lemma 3.3, p.239]), we state our theorem as the following corollary. 
Corollary. Let $\alpha>0$ and let $f$ be an analytic function on $D$. Then

(I) $f \in B M O A$ if and only if $\left|f^{[\alpha]}(z)\right|^{2}\left(1-|z|^{2}\right)^{2 \alpha-1} d A(z)$ is a Carleson measure on $D$.

(II) $f \in V M O A$ if and only if $\left|f^{[\alpha]}(z)\right|^{2}\left(1-|z|^{2}\right)^{2 \alpha-1} d A(z)$ is a vanishing Carleson measure on $D$.

\section{REFERENCES}

[1] J.B. Garnett, Bounded analytic functions (Academic Press, New York, 1981).

[2] S.C. Power, 'Vanishing Carleson measures', Bull. London Math. Soc. 12 (1980), 207-210.

[3] D. Sarason, Function theory on the unit circle, Lecture Notes (Conference at Virginia Polytechnic and State University, Blacksburg, Virginia, 1978).

[4] A.L. Shields and D.L. Williams, 'Bounded projections, duality and multipliers in spaces of analytic functions', Trans. Amer. Math. Soc. 162 (1971), 287-302.

[5] K. Stroethoff, 'Besov-type characterisations for the Bloch space', Bull. Austral. Math. Soc. 39 (1989), 405-420.

Department of Mathematics

Hangehou University

Hangzhou, Zhejiang

People's Republic of China 